\title{
The Selberg integral and Young books (Extended Abstract)
}

\author{
Jang Soo Kim 1fk and Suho $\mathrm{Oh}^{2}$ \\ ${ }^{1}$ Department of Mathematics, Sungkyunkwan University, Suwon 440-746, South Korea \\ ${ }^{2}$ University of Michigan, Ann Arbor, MI, USA
}

\begin{abstract}
The Selberg integral is an important integral first evaluated by Selberg in 1944. Stanley found a combinatorial interpretation of the Selberg integral in terms of permutations. In this paper, new combinatorial objects "Young books" are introduced and shown to have a connection with the Selberg integral. This connection gives an enumeration formula for Young books. It is shown that special cases of Young books become standard Young tableaux of various shapes: shifted staircases, squares, certain skew shapes, and certain truncated shapes. As a consequence, enumeration formulas for standard Young tableaux of these shapes are obtained.

Résumé. L'intégrale de Selberg est une partie intégrante importante abord évalué par Selberg en 1944. Stanley a trouvé une interprétation combinatoire de la Selberg aide en permutations. Dans ce papier, une nouvelle objets combinatoires "livre de Young" sont introduits et présentés à avoir un lien avec l'intégrale de Selberg. Cette connexion donne énumération formules pour les livres de Young. Il est démontré que spécial cas de livres de Young deviennent tableaux standards de Young de divers formes: escaliers décalées, places, certaines formes gauches et certaines formes tronquées. En conséquence, l'énumération des formules pour tableaux standards de Young de ces formes sont obtenues.
\end{abstract}

Keywords: Selberg integral, standard Young tableau, hook length formula

\section{Introduction}

The Selberg integral is the following integral first evaluated by Selberg [Sel44] in 1944:

$$
\begin{aligned}
S_{n}(\alpha, \beta, \gamma) & =\int_{0}^{1} \cdots \int_{0}^{1} \prod_{i=1}^{n} x_{i}^{\alpha-1}\left(1-x_{i}\right)^{\beta-1} \prod_{1 \leq i<j \leq n}\left|x_{i}-x_{j}\right|^{2 \gamma} d x_{1} \cdots d x_{n} \\
& =\prod_{j=1}^{n} \frac{\Gamma(\alpha+(j-1) \gamma) \Gamma(\beta+(j-1) \gamma) \Gamma(1+j \gamma)}{\Gamma(\alpha+\beta+(n+j-2) \gamma) \Gamma(1+\gamma)}
\end{aligned}
$$

where $n$ is a positive integer and $\alpha, \beta, \gamma$ are complex numbers such that $\operatorname{Re}(\alpha)>0, \operatorname{Re}(\beta)>0$, and $\operatorname{Re}(\gamma)>-\min \{1 / n, \operatorname{Re}(\alpha) /(n-1), \operatorname{Re}(\beta) /(n-1)\}$. We refer the reader to Forrester and Warnaar's exposition [FW08] for the history and importance of the Selberg integral.

In [Sta11, Exercise 1.10 (b)] Stanley gives a combinatorial interpretation of the Selberg integral when the exponents $\alpha-1, \beta-1$ and $2 \gamma$ are nonnegative integers by introducing certain permutations. In this paper we define "Selberg books" which are essentially a graphical representation of these permutations. We then define "Young books" which are special Selberg books. Young books are a generalization of both of shifted Young tableaux of staircase shape and standard Young tableaux of square shape. We show that there is a simple relation between the number of Selberg books and the number of Young books by finding generating functions for both objects. Using this relation and the Selberg integral formula, we obtain some enumeration results on standard Young tableaux of certain shapes.

\footnotetext{
${ }^{*}$ The first author was partially supported by Basic Science Research Program through the National Research Foundation of Korea (NRF) funded by the Ministry of Education (NRF-2013R1A1A2061006).
} 
The rest of this paper is organized as follows. In Section 2 we review Stanley's combinatorial interpretation of the Selberg integral. In Section 3 we define Selberg books and Young books in a simple form which are related to the Selberg integral when $\alpha=\beta=1$. We show that there is a simple relation between their cardinalities by finding generating functions for them. Using this relation and (1) we obtain a formula for the number of Young books. In Section 4 we define Selberg books and Young books in a complete form which are related to the Selberg integral without restriction. Results in the previous section are extended here. As consequences we obtain enumeration formulas for standard Young tableaux of a "truncated" shape which is obtained from a rectangle by removing a staircase from the southwest corner, and a certain skew shape which is obtained by attaching two such truncated shapes. Using generating functions, we find another integral expression for the Selberg integral. In Section 5 we consider generalized Selberg books. We find a formula for the number of standard Young tableaux of a truncated shape, which is more general than two truncated shapes considered by Panova [Pan12]. In Section 6 we propose some open problems which will lead to a combinatorial proof of the Selberg integral formula.

\section{Stanley's combinatorial interpretation}

In this section we review Stanley's combinatorial interpretation of the Selberg integral in terms of probability when $r=\alpha-1, s=\beta-1$ and $m=2 \gamma$ are nonnegative integers.

Let $A(n, r, s, m)$ be the following set of letters

$$
\begin{aligned}
A(n, r, s, m)=\left\{x_{i}: 1 \leq i \leq n\right\} \cup & \left\{a_{i j}^{(k)}: 1 \leq i<j \leq n, 1 \leq k \leq m\right\} \\
& \cup\left\{b_{i}^{(k)}: 1 \leq i \leq n, 1 \leq k \leq r\right\} \cup\left\{c_{i}^{(k)}: 1 \leq i \leq n, 1 \leq k \leq s\right\} .
\end{aligned}
$$

A permutation of $A(n, r, s, m)$ is called a Selberg permutation if the following conditions hold:

- $x_{1}, x_{2}, \ldots, x_{n}$ are in this order,

- $a_{i j}^{(k)}$ is between $x_{i}$ and $x_{j}$ for $1 \leq i<j \leq n$ and $1 \leq k \leq m$,

- $b_{i}^{(k)}$ is before $x_{i}$ for $1 \leq i \leq n$ and $1 \leq k \leq r$, and

- $c_{i}^{(k)}$ is after $x_{i}$ for $1 \leq i \leq n$ and $1 \leq k \leq s$.

Let $\operatorname{SP}(n, r, s, m)$ denote the set of Selberg permutations of $A(n, r, s, m)$. Then we have the following combinatorial interpretation for the Selberg integral, see [Sta11, Exercise 1.10 (b)].

Proposition 2.1. We have

$$
\int_{0}^{1} \cdots \int_{0}^{1} \prod_{i=1}^{n} x_{i}^{r}\left(1-x_{i}\right)^{s} \prod_{1 \leq i<j \leq n}\left|x_{i}-x_{j}\right|^{m} d x_{1} \cdots d x_{n}=\frac{n !|\operatorname{SP}(n, r, s, m)|}{((r+s+1) n+m n(n-1) / 2) !} .
$$

For a nonnegative integer $n$, we define

$$
n ! !=\prod_{j=0}^{\lfloor(n-1) / 2\rfloor}(n-2 j) .
$$

In other words,

$$
(2 k) ! !=(2 k)(2 k-2) \cdots 2, \quad(2 k-1) ! !=(2 k-1)(2 k-3) \cdots 1 .
$$

By (1), Proposition 2 and the facts $\Gamma(1+n)=n !$ and $\Gamma\left(\frac{1}{2}+n\right)=\frac{(2 n-1) ! !}{2^{n}} \sqrt{\pi}$, we obtain a formula for the number of Selberg permutations.

Proposition 2.2. We have

$$
|\mathrm{SP}(n, r, s, m)|=\frac{2^{n}((r+s+1) n+m n(n-1) / 2) !}{n !} \prod_{j=1}^{n} \frac{(j m) ! !(2 r+(j-1) m) ! !(2 s+(j-1) m) ! !}{m ! !(2 r+2 s+2+(n+j-2) m) ! !} .
$$




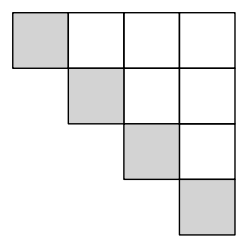

Fig. 1: A shifted staircase of size 4. The diagonal cells are shaded.

\begin{tabular}{|l|l|l|}
\hline 1 & 5 & 2 \\
\hline \multirow{2}{*}{6} & 6 & 8 \\
\cline { 2 - 3 } & & 9 \\
\cline { 2 - 3 }
\end{tabular}

\begin{tabular}{|l|l|l|}
\hline 1 & 4 & 3 \\
\hline \multirow{2}{*}{} & 6 & 7 \\
\cline { 2 - 3 } & 9 \\
\cline { 2 - 3 }
\end{tabular}

Fig. 2: A (3, 2)-Selberg book. The diagonal cells are shaded.

\section{$3(n, m)$-Selberg books and $(n, m)$-Young books}

In this section we define $(n, m)$-Selberg books which are in natural bijection with Selberg permutations $\mathrm{SB}(n, r, s, m)$ when $r=s=0$. We then define $(n, m)$-Young books which are $(n, m)$-Selberg books with an additional condition. In the next section we will consider more general Selberg books and Young books which are related to $\mathrm{SB}(n, r, s, m)$ for nonnegative integers $r$ and $s$.

The shifted staircase of size $n$ is the shifted partition $(n, n-1, \ldots, 1)$. The cell in the $i$ th row and $i$ th column is called the ith diagonal cell. We will identify the shifted staircase of size $n$ with its shifted Ferrers diagram as shown in Figure 1.

Definition 3.1. Let $\lambda^{(1)}, \lambda^{(2)}, \ldots, \lambda^{(m)}$ be shifted staircases of size $n$. We identify the $i$ th diagonal cells of $\lambda^{(1)}, \lambda^{(2)}, \ldots, \lambda^{(m)}$ for each $1 \leq i \leq n$. We call $\lambda^{(i)}$ the ith page. An $(n, m)$-Selberg book is a filling of the $m$-tuple $\left(\lambda^{(1)}, \lambda^{(2)}, \ldots, \lambda^{(m)}\right)$ with integers $1,2, \ldots, n+m\left(\begin{array}{l}n \\ 2\end{array}\right)$ such that in each page the integer in the $i$ th row and $j$ th column with $i \neq j$ is bigger than the integer in the $i$ th diagonal cell and smaller than the integer in the $j$ th diagonal cell. Let $\mathrm{SB}(n, m)$ be the set of $(n, m)$-Selberg books.

See Figure 2 for an example of $(n, m)$-Selberg book.

There is a natural bijection between $\operatorname{SB}(n, m)$ and $\operatorname{SP}(n, 0,0, m)$ as follows. For $B \in \operatorname{SB}(n, m)$, define the corresponding permutation $\pi=\pi_{1} \pi_{2} \ldots \pi_{n+m\left(\begin{array}{c}n \\ 2\end{array}\right)}$ by

$$
\pi_{\ell}= \begin{cases}x_{i}, & \text { if } B \text { has } \ell \text { in the } i \text { th diagonal cell, } \\ a_{i j}^{(k)}, & \text { if } B \text { has } \ell \text { in the } i \text { th row and } j \text { th column of the } k \text { th shifted staircase with } i \neq j .\end{cases}
$$

The permutation corresponding to the Selberg book in Figure 2 is

$$
x_{1} a_{13}^{(1)} a_{13}^{(2)} a_{12}^{(2)} a_{12}^{(1)} x_{2} a_{23}^{(2)} a_{23}^{(1)} x_{3} .
$$

Thus, by Proposition 2.2 with $r=s=0$, we obtain a formula for $|\mathrm{SB}(n, m)|$.

Proposition 3.1. We have

$$
|\mathrm{SB}(n, m)|=\frac{2^{n}(n+m n(n-1) / 2) !}{n ! m ! !^{n}} \prod_{j=1}^{n} \frac{((j-1) m) ! !^{2}(j m) ! !}{(2+(n+j-2) m) ! !} .
$$

Definition 3.2. An $(n, m)$-Young book is an $(n, m)$-Selberg book with the additional condition that for each shifted staircase the integers are increasing along each row and column. Let $\mathrm{YB}(n, m)$ be the set of $(n, m)$-Young books.

Note that an $(n, 1)$-Young book is a just shifted standard Young tableaux of shifted staircase shape. By attaching the two shifted staircases along the diagonal cells after flipping over the second shifted staircase, an $(n, 2)$-Young book can be thought of as a standard Young tableaux of square shape $\left(n^{n}\right)$, see Figure 3 . 

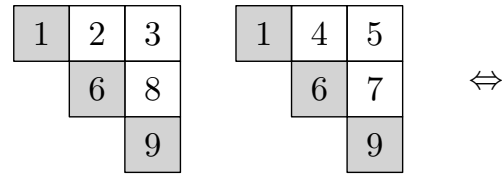

\begin{tabular}{|l|l|l|}
\hline 1 & 2 & 3 \\
\hline 4 & 6 & 8 \\
\hline 5 & 7 & 9 \\
\hline
\end{tabular}

Fig. 3: The correspondence between $(n, 2)$-Selberg books and standard Young tableaux of square shape $\left(n^{n}\right)$. The diagonal cells are shaded.

For the rest of this section we will find a simple relation between the cardinalities of $\operatorname{SB}(n, m)$ and $\mathrm{YB}(n, m)$.

We define $\operatorname{SB}\left(n, m ; d_{1}, \ldots, d_{n-1}\right)$ and $\mathrm{YB}\left(n, m ; d_{1}, \ldots, d_{n-1}\right)$ to be, respectively, the set of $(n, m)$ Selberg books and the set of $(n, m)$-Young books such that the entries $a_{1}, a_{2}, \ldots, a_{n}$ in the diagonal cells satisfy $d_{i}=a_{i+1}-a_{i}-1$ for $i=1,2, \ldots, n-1$. Note that since we always have $a_{1}=1$ and $a_{n}=$ $n+m\left(\begin{array}{c}n \\ 2\end{array}\right)$, the numbers $d_{1}, \ldots, d_{n-1}$ determine $a_{1}, a_{2}, \ldots, a_{n}$, and vice versa.

Proposition 3.2. For nonnegative integers $n$ and $m$, we have

$$
\sum_{d_{1}, \ldots, d_{n-1} \geq 0}\left|\operatorname{SB}\left(n, m ; d_{1}, \ldots, d_{n-1}\right)\right| \frac{t_{1}^{d_{1}} \ldots t_{n-1}^{d_{n-1}}}{d_{1} ! \ldots d_{n-1} !}=\prod_{1 \leq i<j \leq n}\left(t_{i}+t_{i+1}+\cdots+t_{j-1}\right)^{m} .
$$

Proof. We define a reduced ( $n, m)$-Selberg book to be a filling of an $m$-tuple of shifted staircases of size $n$ with integers $1,2, \ldots, n-1$ repetition allowed such that the diagonal cells remain empty and a non-diagonal cell in the $i$ th row and $j$ th column is filled with an integer $k$ satisfying $i \leq k<j$. Let $\operatorname{RSB}\left(n, m ; d_{1}, \ldots, d_{n-1}\right)$ denote the set of reduced $(n, m)$-Selberg books with $d_{1} 1$ 's, $d_{2} 2$ 's, and so on. By definition we have

$$
\sum_{d_{1}, \ldots, d_{n-1} \geq 0}\left|\operatorname{RSB}\left(n, m ; d_{1}, \ldots, d_{n-1}\right)\right| t_{1}^{d_{1}} \ldots t_{n-1}^{d_{n-1}}=\prod_{1 \leq i<j \leq n}\left(t_{i}+t_{i+1}+\cdots+t_{j-1}\right)^{m} .
$$

Let $B \in \mathrm{SB}\left(n, m ; d_{1}, \ldots, d_{n-1}\right)$. Then the entries $a_{1}, \ldots, a_{n}$ in the diagonal cells of $B$ satisfy $d_{i}=$ $a_{i+1}-a_{i}-1$. Let $B^{\prime}$ be the reduced $(n, m)$-Selberg book obtained from $B$ by replacing the $d_{i}$ integers $a_{i}+1, a_{i}+2, \ldots, a_{i+1}-1$ in $B$ with $i$ 's for each $i=1,2, \ldots, n-1$. It is easy to see that the map $B \mapsto B^{\prime}$ is 1 -to- $d_{1} ! \ldots d_{n-1}$ !, which finishes the proof.

Postnikov [Pos09] showed that

$$
\sum_{d_{1}, \ldots, d_{n-1} \geq 0}\left|\mathrm{YB}\left(n, 1 ; d_{1}, \ldots, d_{n-1}\right)\right| \frac{t_{1}^{d_{1}} \ldots t_{n-1}^{d_{n-1}}}{d_{1} ! \ldots d_{n-1} !}=\prod_{1 \leq i<j \leq n} \frac{t_{i}+t_{i+1}+\cdots+t_{j-1}}{j-i} .
$$

Theorem 3.3. We have

$$
\begin{aligned}
\left|\mathrm{SB}\left(n, m ; d_{1}, \ldots, d_{n-1}\right)\right| & =(1 ! 2 ! \cdots(n-1) !)^{m} \cdot\left|\mathrm{YB}\left(n, m ; d_{1}, \ldots, d_{n-1}\right)\right|, \\
|\mathrm{SB}(n, m)| & =(1 ! 2 ! \cdots(n-1) !)^{m} \cdot|\mathrm{YB}(n, m)| .
\end{aligned}
$$

Proof. Since (4) is obtained from (3) by summing over all $d_{1}, \ldots, d_{n-1}$, it suffices to prove (3). By Proposition 3.2 and (2), we have

$$
\left|\mathrm{SB}\left(n, 1 ; d_{1}, \ldots, d_{n-1}\right)\right|=1 ! 2 ! \cdots(n-1) ! \cdot\left|\mathrm{YB}\left(n, 1 ; d_{1}, \ldots, d_{n-1}\right)\right| .
$$

For a set $X$ of $\left(\begin{array}{l}n \\ 2\end{array}\right)$ integers, let $\mathrm{SB}_{X}\left(n, 1 ; d_{1}, \ldots, d_{n-1}\right)$ to be the set of fillings of a shifted staircase of size $n$ with integers in $X \cup\left\{d_{1}, \ldots, d_{n-1}\right\}$ so that the $i$ th diagonal cell is filled with $d_{i}$ and a non-diagonal cell in the $i$ th row and $j$ th column is filled with an integer $k$ satisfying $d_{i}<k<d_{j}$. In other words, if we replace the $i$ th smallest integer of $X \cup\left\{d_{1}, \ldots, d_{n-1}\right\}$ in $B \in \mathrm{SB}_{X}\left(n, 1 ; d_{1}, \ldots, d_{n-1}\right)$ with $i$ for $i=1,2, \ldots,\left(\begin{array}{c}n+1 \\ 2\end{array}\right)$, then we get an element in $\operatorname{SB}\left(n, 1 ; d_{1}^{\prime}, \ldots, d_{n-1}^{\prime}\right)$, where $d_{i}^{\prime}$ is the integer for which 
$d_{i}$ is the $d_{i}^{\prime}$ th smallest integer of $X \cup\left\{d_{1}, \ldots, d_{n-1}\right\}$. By considering the each shifted staircase separately we get

$$
\left|\mathrm{SB}\left(n, m ; d_{1}, \ldots, d_{n-1}\right)\right|=\sum_{X_{1}, \ldots, X_{m}} \prod_{i=1}^{m}\left|\mathrm{SB}_{X_{i}}\left(n, 1 ; d_{1}, \ldots, d_{n-1}\right)\right|,
$$

where the sum is over all subsets $X_{1}, \ldots, X_{m}$ of $\left\{1,2, \ldots, m\left(\begin{array}{l}n \\ 2\end{array}\right)\right\}$ such that $\left|X_{i}\right|=\left(\begin{array}{l}n \\ 2\end{array}\right)$ and

$$
X_{1} \cup \cdots \cup X_{m}=\left\{1,2, \ldots, m\left(\begin{array}{c}
n \\
2
\end{array}\right)\right\} \backslash\left\{d_{1}, \ldots, d_{n-1}\right\} .
$$

Similarly we can define $\operatorname{YB}_{X}\left(n, 1 ; d_{1}, \ldots, d_{n-1}\right)$ and obtain

$$
\left|\mathrm{YB}\left(n, m ; d_{1}, \ldots, d_{n-1}\right)\right|=\sum_{X_{1}, \ldots, X_{m}} \prod_{i=1}^{m}\left|\mathrm{YB}_{X_{i}}\left(n, 1 ; d_{1}, \ldots, d_{n-1}\right)\right| .
$$

For given $X_{i}$, we have

$$
\begin{aligned}
\left|\mathrm{SB}_{X_{i}}\left(n, 1 ; d_{1}, \ldots, d_{n-1}\right)\right| & =\left|\operatorname{SB}\left(n, 1 ; d_{1}^{\prime}, \ldots, d_{n-1}^{\prime}\right)\right|, \\
\left|\mathrm{YB}_{X_{i}}\left(n, 1 ; d_{1}, \ldots, d_{n-1}\right)\right| & =\left|\mathrm{YB}\left(n, 1 ; d_{1}^{\prime}, \ldots, d_{n-1}^{\prime}\right)\right|,
\end{aligned}
$$

for the same $d_{1}^{\prime}, \ldots, d_{n-1}^{\prime}$. Thus by (5) we have

$$
\left|\mathrm{SB}_{X_{i}}\left(n, 1 ; d_{1}, \ldots, d_{n-1}\right)\right|=1 ! 2 ! \cdots(n-1) ! \cdot\left|\mathrm{YB}_{X_{i}}\left(n, 1 ; d_{1}, \ldots, d_{n-1}\right)\right| .
$$

Applying the above equation to (6) and (7) we get (3).

By Proposition 3.2 and (3) we obtain the following generalization of Postnikov's result (2).

\section{Corollary 3.4.}

$$
\sum_{d_{1}, \ldots, d_{n-1} \geq 0}\left|\mathrm{YB}\left(n, m ; d_{1}, \ldots, d_{n-1}\right)\right| \frac{t_{1}^{d_{1}} \ldots t_{n-1}^{d_{n-1}}}{d_{1} ! \ldots d_{n-1} !}=\left(\prod_{1 \leq i<j \leq n} \frac{t_{i}+t_{i+1}+\cdots+t_{j-1}}{j-i}\right)^{m} .
$$

We note that Corollary 3.4 can also be proved directly from (2) using (7).

By Proposition 3.1 and (4) we get the number of $(n, m)$-Young books.

Corollary 3.5. We have

$$
|\mathrm{YB}(n, m)|=\frac{2^{n}(n+m n(n-1) / 2) !}{n ! m ! !^{n}} \prod_{j=1}^{n} \frac{((j-1) m) ! !^{2}(j m) ! !}{(j-1) !^{m}(2+(n+j-2) m) ! !} .
$$

If $m=1$ in Corollary 3.5 , then we get the hook length formula for the number of shifted standard Young tableaux of staircase shape $(n, n-1, \ldots, 1)$. If $m=2$ in Corollary 3.5 the we get the hook length formula for the number of standard Young tableaux of square shape $(n, n, \ldots, n)$. This gives a semi-combinatorial proof of the Selberg integral for $\alpha=\beta=1$ and $\gamma \in\{1 / 2,1\}$.

\section{4 ( $n, \mathbf{r}, \mathbf{s})$-Selberg books and $(n, \mathbf{r}, \mathbf{s})$-Young books}

In this section we will define $(n, \mathbf{r}, \mathbf{s})$-Selberg books and $(n, \mathbf{r}, \mathbf{s})$-Young books which are related to $\mathrm{SB}(n, r, s, m)$.

An $(n, r, s)$-staircase is the diagram obtained from the $(r+n) \times(n+s)$ rectangle by removing the cells below the diagonal cells, where the cell in the $(i+r)$ th row and $i$ th column is called the $i$ th diagonal cell. A truncated $(n, r, s)$-staircase is the diagram obtained from an $(n, r, s)$-staircase by removing the $r \times s$ rectangle at the upper right corner. See Figure 4 

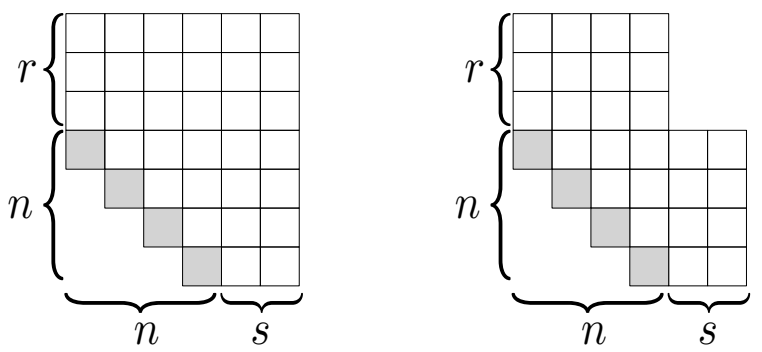

Fig. 4: An $(n, r, s)$-staircase on the left and a truncated $(n, r, s)$-staircase on the right. The diagonal cells are shaded.

Definition 4.1. Let $\mathbf{r}=\left(r_{1}, \ldots, r_{m}\right)$ and $\mathbf{s}=\left(s_{1}, \ldots, s_{m}\right)$ be two sequences of nonnegative integers with $r_{1}+\cdots+r_{m}=r$ and $s_{1}+\cdots+s_{m}=s$. For $1 \leq i \leq m$, let $\lambda^{(i)}$ be a truncated $\left(n, r_{i}, s_{i}\right)$-staircase. We identify the $i$ th diagonal cells of $\lambda^{(1)}, \lambda^{(2)}, \ldots, \lambda^{(m)}$ for each $1 \leq i \leq n$. We call $\lambda^{(i)}$ the ith page. A truncated $(n, \mathbf{r}, \mathbf{s})$-Selberg book is a filling of $\left(\lambda^{(1)}, \ldots, \lambda^{(m)}\right)$ with $1,2, \ldots,(r+s+1) n+m\left(\begin{array}{c}n \\ 2\end{array}\right)$ such that the integer in a non-diagonal cell of each page is bigger than the integer in the diagonal cell of the same row and smaller than the integer in the diagonal cell of the same column. Let $\mathrm{SB}^{-}(n, \mathbf{r}, \mathbf{s})$ denote the set of truncated $(n, \mathbf{r}, \mathbf{s})$-Selberg books.

The bijection between $\operatorname{SB}(n, m)$ and $\operatorname{SP}(n, 0,0, m)$ in Section 3 can easily be extended to a bijection between $\mathrm{SB}^{-}(n, \mathbf{r}, \mathbf{s})$ and $\mathrm{SP}(n, r, s, m)$. Notice that the cardinality of $\mathrm{SB}^{-}(n, \mathbf{r}, \mathbf{s})$ depends only on $n, r, s$. Thus we obtain the following proposition.

Proposition 4.1. Let $\mathbf{r}=\left(r_{1}, \ldots, r_{m}\right)$ and $\mathbf{s}=\left(s_{1}, \ldots, s_{m}\right)$ be two sequences of nonnegative integers with $r_{1}+\cdots+r_{m}=r$ and $s_{1}+\cdots+s_{m}=s$. Then

$$
\left|\mathrm{SB}^{-}(n, \mathbf{r}, \mathbf{s})\right|=|\mathrm{SP}(n, r, s, m)| .
$$

Now we define $(n, \mathbf{r}, \mathbf{s})$-Selberg books. Let $\mathbf{r}=\left(r_{1}, \ldots, r_{m}\right)$ and $\mathbf{s}=\left(s_{1}, \ldots, s_{m}\right)$ be two sequences of nonnegative integers with $r_{1}+\cdots+r_{m}=r$ and $s_{1}+\cdots+s_{m}=s$. For $1 \leq i \leq m$, let $\lambda^{(i)}$ be an $\left(n, r_{i}, s_{i}\right)$-staircase. We identify the diagonal cells of $\lambda^{(i)}$ for all $1 \leq i \leq m$. An $(n, \mathbf{r}, \mathbf{s})$-Selberg book is a filling of $\left(\lambda^{(1)}, \ldots, \lambda^{(m)}\right)$ with $1,2, \ldots,(r+s+1) n+m\left(\begin{array}{c}n \\ 2\end{array}\right)+\sum_{i=1}^{m} r_{i} s_{i}$ such that in each page the integer in a non-diagonal cell is bigger than the integer in the diagonal cell of the same row and smaller than the integer in the diagonal cell of the same column. Let $\operatorname{SB}(n, \mathbf{r}, \mathbf{s})$ denote the set of $(n, \mathbf{r}, \mathbf{s})-\operatorname{Selberg}$ books.

Proposition 4.2. Let $\mathbf{r}=\left(r_{1}, \ldots, r_{m}\right)$ and $\mathbf{s}=\left(s_{1}, \ldots, s_{m}\right)$ be two sequences of nonnegative integers with $r_{1}+\cdots+r_{m}=r$ and $s_{1}+\cdots+s_{m}=s$. Then

$$
|\mathrm{SB}(n, \mathbf{r}, \mathbf{s})|=\left|\mathrm{SB}^{-}(n, \mathbf{r}, \mathbf{s})\right| \frac{\left((r+s+1) n+m\left(\begin{array}{c}
n \\
2
\end{array}\right)+\sum_{i=1}^{m} r_{i} s_{i}\right) !}{\left((r+s+1) n+m\left(\begin{array}{c}
n \\
2
\end{array}\right)\right) !} .
$$

We define an $(n, \mathbf{r}, \mathbf{s})$-Young book to be an $(n, \mathbf{r}, \mathbf{s})$-Selberg book such that in each page the entries are increasing from left to right and from top to bottom. Let $\mathrm{YB}(n, \mathbf{r}, \mathbf{s})$ denote the set of $(n, \mathbf{r}, \mathbf{s})$-Young books. We also define $\operatorname{SB}\left(n, \mathbf{r}, \mathbf{s} ; d_{0}, d_{1}, \ldots, d_{n}\right)$ and $\mathrm{YB}\left(n, \mathbf{r}, \mathbf{s} ; d_{0}, d_{1}, \ldots, d_{n}\right)$ to be, respectively, the set of $(n, \mathbf{r}, \mathbf{s})$-Selberg books and the set of $(n, \mathbf{r}, \mathbf{s})$-Young books whose diagonal entries $a_{1}, \ldots, a_{n}$ satisfy $d_{i}=a_{i+1}-a_{i}-1$ for $i=0,1,2, \ldots, n$, where $a_{0}=1$ and $a_{n+1}=(r+s+1) n+m\left(\begin{array}{c}n \\ 2\end{array}\right)+\sum_{i=1}^{m} r_{i} s_{i}$.

The following lemma is an immediate consequence of the definition of Selberg books and Young books.

Lemma 4.3. Suppose that $d_{1}, d_{2}, \ldots, d_{n-1}$ be a sequence of nonnegative integer such that $d_{k+1}=$ $1, d_{k+2}=2, \ldots, d_{k+\ell-1}=\ell-1$ for some $k, \ell \geq 0$. Then, for any $B \in \mathrm{YB}\left(n, 1 ; d_{1}, d_{2}, \ldots, d_{n-1}\right)$, the entries in rows $k+1, k+2, \ldots, k+\ell$ and columns $k+1, k+2, \ldots, k+\ell$ are completely determined by $d_{1}, \ldots, d_{n-1}$. More precisely, for $1 \leq i, j \leq \ell$, if $x$ is the entry in the $(k+1)$ st diagonal cell, which is determined by $d_{1}, \ldots, d_{n-1}$, then the entry in row $k+i$ and column $k+j$ is $x+\left(\begin{array}{c}j-1 \\ 2\end{array}\right)+i$.

Moreover, if $B \in \mathrm{SB}\left(n, 1 ; d_{1}, d_{2}, \ldots, d_{n-1}\right)$ and $x$ is the entry in the $(k+1)$ st diagonal cell, then the entries in column $k+j$ and in rows $k+1, k+2, \ldots, k+j-1$ form a permutation of $x+\left(\begin{array}{c}j-1 \\ 2\end{array}\right)+1, x+$ $\left(\begin{array}{c}j-1 \\ 2\end{array}\right)+2, \ldots, x+\left(\begin{array}{c}j-1 \\ 2\end{array}\right)+j-1$. 

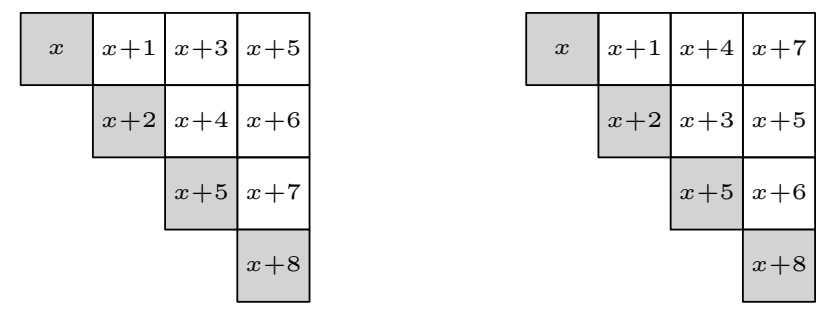

Fig. 5: The diagram on the left shows the typical form of the entries in rows $k+1, k+2, \ldots, k+\ell$ and columns $k+1, k+2, \ldots, k+\ell$ of $B \in \mathrm{YB}\left(n, 1 ; d_{1}, d_{2}, \ldots, d_{n-1}\right)$ when $d_{k+1}=1, d_{k+2}=2, \ldots, d_{k+\ell-1}=\ell-1$. The diagram on the right shows that, in the case of $B \in \mathrm{SB}\left(n, 1 ; d_{1}, d_{2}, \ldots, d_{n-1}\right)$, for $1 \leq j \leq \ell$, the non-diagonal entries in column $k+j$ and below row $k$ are obtained by permuting those in the same cells of the diagram on the left. In this example, we have $\ell=4$.
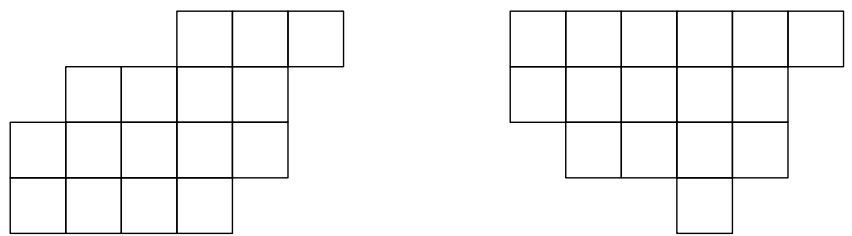

Fig. 6: The skew shape $\lambda / \mu$ on the left and the truncated shape $\lambda \backslash \mu$ on the right for $\lambda=(6,5,5,4)$ and $\mu=(3,1)$.

Figure 5 illustrates the situation in Lemma 4.3 .

Proposition 4.4. Let $\mathbf{r}=\left(r_{1}, \ldots, r_{m}\right)$ and $\mathbf{s}=\left(s_{1}, \ldots, s_{m}\right)$ be sequences of nonnegative integers. Then we have

$$
\left|\mathrm{SB}\left(n, \mathbf{r}, \mathbf{s} ; d_{0}, d_{1}, \ldots, d_{n}\right)\right|=\left|\mathrm{YB}\left(n, \mathbf{r}, \mathbf{s} ; d_{0}, d_{1}, \ldots, d_{n}\right)\right| \prod_{i=1}^{m} \frac{1 ! 2 ! \cdots\left(n+r_{i}+s_{i}-1\right) !}{1 ! 2 ! \cdots\left(r_{i}-1\right) ! 1 ! 2 ! \cdots\left(s_{i}-1\right) !} .
$$

By Propositions 2.2, 4.1, 4.2, and 4.4, we get a formula for $|\mathrm{YB}(n, \mathbf{r}, \mathbf{s})|$.

Theorem 4.5. Let $\mathbf{r}=\left(r_{1}, \ldots, r_{m}\right)$ and $\mathbf{s}=\left(s_{1}, \ldots, s_{m}\right)$ be sequences of nonnegative integers with $r_{1}+\cdots+r_{m}=r$ and $s_{1}+\cdots+s_{m}=s$. Then

$$
\begin{aligned}
|\mathrm{YB}(n, \mathbf{r}, \mathbf{s})|=\left((r+s+1) n+m\left(\begin{array}{l}
n \\
2
\end{array}\right)+\right. & \left.\sum_{i=1}^{m} r_{i} s_{i}\right) ! \prod_{i=1}^{m} \frac{1 ! 2 ! \cdots\left(r_{i}-1\right) ! 1 ! 2 ! \cdots\left(s_{i}-1\right) !}{1 ! 2 ! \cdots\left(n+r_{i}+s_{i}-1\right) !} \\
& \times \frac{2^{n}}{n !} \prod_{j=1}^{n} \frac{(j m) ! !(2 r+(j-1) m) ! !(2 s+(j-1) m) ! !}{m ! !(2 r+2 s+2+(n+j-2) m) ! !}
\end{aligned}
$$

For two partitions $\lambda=\left(\lambda_{1}, \ldots, \lambda_{k}\right)$ and $\mu=\left(\mu_{1}, \ldots, \mu_{\ell}\right)$, the usual skew shape $\lambda / \mu$ is defined to be the set-theoretic difference $\lambda-\mu$ of their Ferrers diagrams. We define the truncated shape $\lambda \backslash \mu$ to be the diagram obtained from the Ferrers diagram of $\lambda$ by removing the $\mu_{i}$ cells from the left in the $(k+1-i)$ th row for $i=1,2, \ldots, \ell$. See Figure 6

Notice that, when $m=1$, an $(n,(r),(s))$-Young book is the same as a standard Young tableau of truncated shape $\lambda \backslash \mu$ for $\lambda=\left((n+s)^{r+n}\right)$ and $\mu=(n-1, n-2, \ldots, 1)$.

Corollary 4.6. The number of standard Young tableaux of truncated shape

$$
\left((n+s)^{r+n}\right) \backslash(n-1, n-2, \ldots, 1)
$$

is

$$
\left((r+s+1) n+\left(\begin{array}{l}
n \\
2
\end{array}\right)+r s\right) ! \frac{2^{n} F(r) F(s)}{n ! F(n+r+s)} \prod_{j=1}^{n} \frac{(j) ! !(2 r+j-1) ! !(2 s+j-1) ! !}{(2 r+2 s+n+j) ! !},
$$

where $F(k)=1 ! 2 ! \cdots(k-1) !$. 

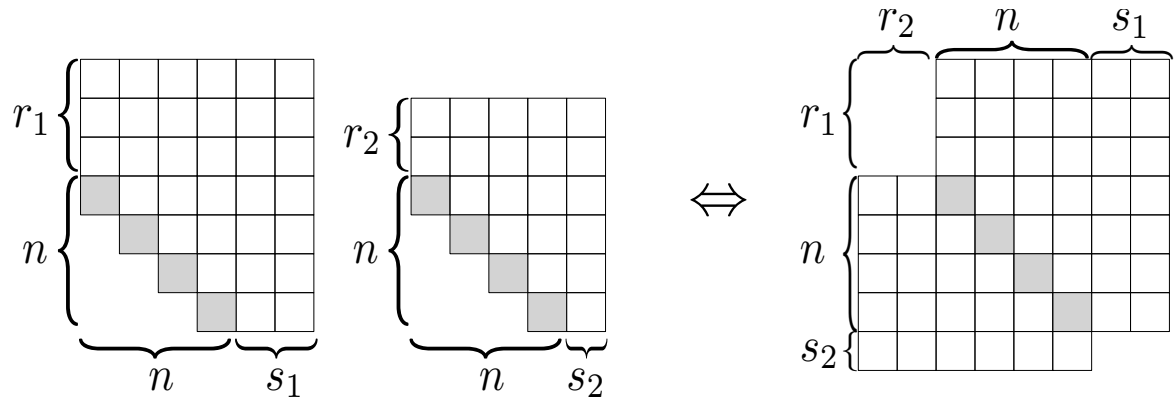

Fig. 7: The skew shape $\lambda / \mu$ on the right is obtained by attaching an $\left(n, r_{1}, r_{2}\right)$-staircase and an $\left(n, r_{2}, s_{2}\right)$-staircase along the diagonal cells. The diagonal cells are shaded and the $\left(n, r_{2}, s_{2}\right)$-staircase is flipped when attached.

When $m=2$, by attaching the two pages along the diagonal cells, an $\left(n,\left(r_{1}, r_{2}\right),\left(s_{1}, s_{2}\right)\right)$-Young book can be thought of as a standard Young tableau of skew shape $\lambda / \mu$ for

$$
\lambda=(\overbrace{r_{2}+n+s_{1}, \ldots, r_{2}+n+s_{1}}^{r_{1}+n}, \overbrace{r_{2}+n, \ldots, r_{2}+n}^{s_{2}}), \quad \mu=(\overbrace{r_{2}, \ldots, r_{2}}^{r_{1}}) .
$$

The Ferrers diagram of $\lambda / \mu$ is drawn on the right in Figure 7.

Corollary 4.7. For the partitions $\lambda$ and $\mu$ in (8), the number of standard Young tableaux of skew shape $\lambda / \mu$ is

$$
\frac{2^{n}\left((r+s) n+n^{2}+r_{1} s_{1}+r_{2} s_{2}\right) ! F\left(r_{1}\right) F\left(r_{2}\right) F\left(s_{1}\right) F\left(s_{2}\right)}{n ! F\left(n+r_{1}+s_{1}\right) F\left(n+r_{2}+s_{2}\right)} \prod_{j=1}^{n} \frac{(2 j) ! !(2 r+2 j-2) ! !(2 s+2 j-2) ! !}{(2 r+2 s+2 n+2 j-2) ! !} .
$$

where $F(k)=1 ! 2 ! \cdots(k-1)$ !.

Remark 4.1. If $\lambda=(7,7,7,7,7,5,5)$ and $\mu=(4,4)$, then the number of standard Young tableaux of skew shape $\lambda / \mu$ has a factor of 9173 . So in general the number of standard Young tableaux of a rectangular shape with two smaller rectangles missing in the northwest corner and in the southeast corner does not seem to have a product formula unless $\lambda$ and $\mu$ are of the form in (8).

By the same arguments in the previous section, one can prove the following two propositions.

Proposition 4.8. Let $\mathbf{r}=\left(r_{1}, \ldots, r_{m}\right)$ and $\mathbf{s}=\left(s_{1}, \ldots, s_{m}\right)$ be sequences of nonnegative integers. Then we have

$$
\begin{aligned}
\sum_{d_{0}, d_{1}, \ldots, d_{n} \geq 0} \mid \mathrm{SB}(n, \mathbf{r}, \mathbf{s} ; & \left.d_{0}, d_{1}, \ldots, d_{n}\right) \mid \frac{t_{0}^{d_{1}} t_{1}^{d_{1}} \ldots t_{n}^{d_{n}}}{d_{0} ! d_{1} ! \ldots d_{n} !} \\
=\prod_{i=1}^{n}\left(t_{0}+\right. & \left.t_{1}+\cdots+t_{i-1}\right)^{r}\left(t_{i}+t_{i+1}+\cdots+t_{n}\right)^{s} \\
& \times \prod_{i=1}^{m}\left(t_{0}+t_{1}+\cdots+t_{n}\right)^{r_{i} s_{i}} \prod_{1 \leq i<j \leq n}\left(t_{i}+t_{i+1}+\cdots+t_{j-1}\right)^{m} .
\end{aligned}
$$

Proposition 4.9. Let $\mathbf{r}=\left(r_{1}, \ldots, r_{m}\right)$ and $\mathbf{s}=\left(s_{1}, \ldots, s_{m}\right)$ be sequences of nonnegative integers. Then we have

$$
\begin{aligned}
& \sum_{d_{0}, d_{1}, \ldots, d_{n} \geq 0} \mid \mathrm{YB}\left(n, \mathbf{r}, \mathbf{s} ; d_{0}, d_{1}, \ldots,\right.\left.d_{n}\right) \mid \frac{t_{0}^{d_{1}} t_{1}^{d_{1}} \ldots t_{n}^{d_{n}}}{d_{0} !_{1} ! \ldots d_{n} !} \\
&=\prod_{i=1}^{n}\left(t_{0}+t_{1}+\cdots+t_{i-1}\right)^{r}\left(t_{i}+t_{i+1}+\cdots+t_{n}\right)^{s} \prod_{1 \leq i<j \leq n}\left(t_{i}+t_{i+1}+\cdots+t_{j-1}\right)^{m} \\
& \times \prod_{i=1}^{m}\left(t_{0}+t_{1}+\cdots+t_{n}\right)^{r_{i} s_{i}} \frac{1 ! 2 ! \cdots\left(r_{i}-1\right) ! 1 ! 2 ! \cdots\left(s_{i}-1\right) !}{1 ! 2 ! \cdots\left(n+r_{i}+s_{i}-1\right) !} .
\end{aligned}
$$


Using Proposition 4.8 we can obtain another integral expression for the Selberg integral. First, note that

$$
\int_{0}^{\infty} x^{n} e^{-x} d x=n !
$$

Thus

$$
|\mathrm{SB}(n, r, s, m)|=\sum_{d_{0}, d_{1}, \ldots, d_{n} \geq 0}\left|\mathrm{SB}\left(n, r, s, m ; d_{0}, d_{1}, \ldots, d_{n}\right)\right|
$$

is equal to

$$
\int_{0}^{\infty} \ldots \int_{0}^{\infty} \sum_{d_{0}, d_{1}, \ldots, d_{n} \geq 0}\left|\mathrm{SB}\left(n, r, s, m ; d_{0}, d_{1}, \ldots, d_{n}\right)\right| \frac{t_{0}^{d_{0}} t_{1}^{d_{1}} \ldots t_{n}^{d_{n}}}{d_{0} ! d_{1} ! \ldots d_{n} !} e^{-t_{0}-t_{1}-\cdots-t_{n}} d t_{0} d t_{1} \ldots d t_{n} .
$$

Using Propositions 2.1 and 4.8 we get the following.

Proposition 4.10. We have

$$
\begin{aligned}
\int_{0}^{\infty} & \ldots \int_{0}^{\infty} \prod_{i=1}^{n}\left(t_{0}+t_{1}+\cdots+t_{i-1}\right)^{r}\left(t_{i}+t_{i+1}+\cdots+t_{n}\right)^{s} \\
& \times \prod_{1 \leq i<j \leq n}\left(t_{i}+t_{i+1}+\cdots+t_{j-1}\right)^{m} e^{-t_{0}-t_{1}-\cdots-t_{n}} d t_{0} d t_{1} \ldots d t_{n} \\
= & \frac{((r+s+1) n+m n(n-1) / 2) !}{n !} \int_{0}^{1} \cdots \int_{0}^{1} \prod_{i=1}^{n} x_{i}^{r}\left(1-x_{i}\right)^{s} \prod_{1 \leq i<j \leq n}\left|x_{i}-x_{j}\right|^{m} d x_{1} \cdots d x_{n} .
\end{aligned}
$$

We note that it is also possible to prove the above proposition using change of variables.

\section{Generalized Selberg books and Young books}

In this section we generalize Selberg books and Young books so that a diagonal cell can be a bigger square.

Let $\mathbf{a}=\left(a_{1}, \ldots, a_{n}\right)$ be a sequence of positive integers. An $(\mathbf{a}, r, s)$-staircase is the diagram obtained from the truncated shape $\lambda \backslash \mu$ by merging the cells in rows

$$
r+a_{1}+\cdots+a_{i-1}+1, r+a_{1}+\cdots+a_{i-1}+2, \ldots, r+a_{1}+\cdots+a_{i-1}+a_{i},
$$

and columns

$$
a_{1}+\cdots+a_{i-1}+1, a_{1}+\cdots+a_{i-1}+2, \ldots, a_{1}+\cdots+a_{i-1}+a_{i},
$$

into a single cell, called the ith diagonal cell, where

$$
\lambda=\left((a+s)^{(r+a)}\right), \quad \mu=\left(\left(a_{1}+\cdots+a_{n-1}\right)^{a_{n}},\left(a_{1}+\cdots+a_{n-2}\right)^{a_{n-1}}, \ldots, a_{1}^{a^{2}}\right) .
$$

We will consider that the $i$ th diagonal cell is contained in every row whose row index is in 10 , and in every column whose column index is in (11). A truncated $(\mathbf{a}, r, s)$-staircase is the diagram obtained from an $(\mathbf{a}, r, s)$-staircase by removing the $r \times s$ rectangle in the northeast corner. See Figure 8

Throughout this section we will use the following notation. Let $\mathbf{a}=\left(a_{1}, \ldots, a_{n}\right), \mathbf{r}=\left(r_{1}, \ldots, r_{m}\right)$ and $\mathbf{s}=\left(s_{1}, \ldots, s_{m}\right)$ be sequences of nonnegative integers with $a_{1}+\cdots+a_{n}=a, r_{1}+\cdots+r_{m}=r$ and $s_{1}+\cdots+s_{m}=s$. Let

$$
\begin{gathered}
N=n+a(r+s)+m \sum_{1 \leq i<j \leq n} a_{i} a_{j}+\sum_{i=1}^{m} r_{i} s_{i}, \\
N^{-}=n+a(r+s)+m \sum_{1 \leq i<j \leq n} a_{i} a_{j} .
\end{gathered}
$$



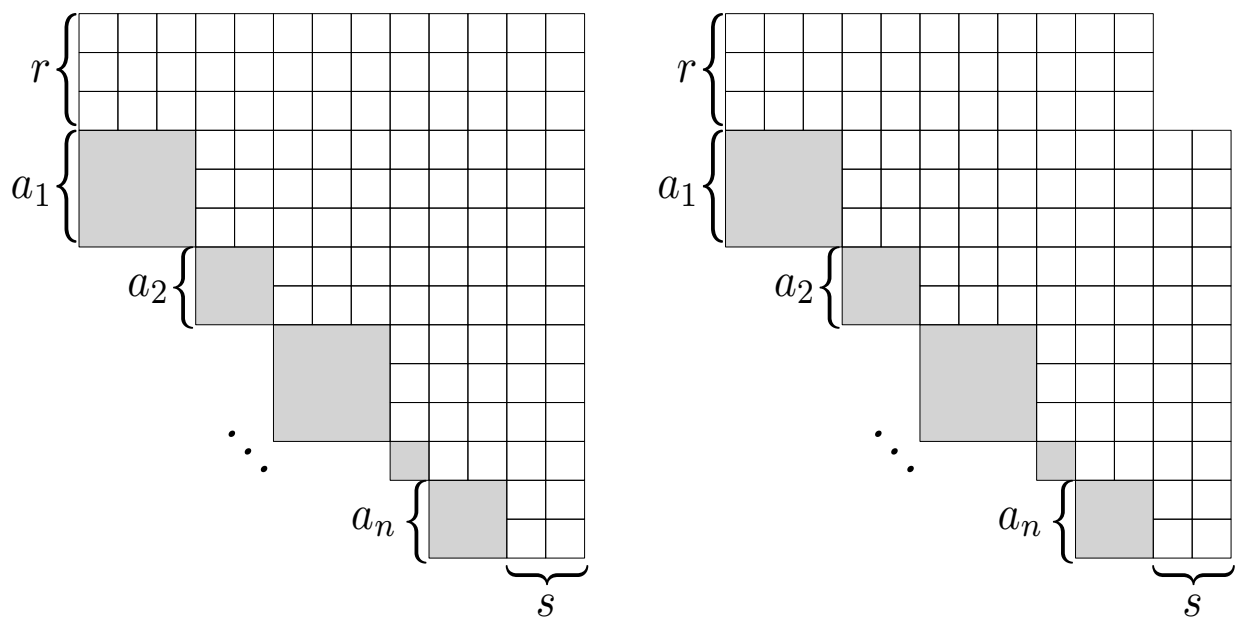

Fig. 8: An $(\mathbf{a}, r, s)$-staircase on the left and a truncated $(\mathbf{a}, r, s)$-staircase on the right. The diagonal cells are shaded.

\begin{tabular}{|c|c|c|c|c|}
\hline 4 & 2 & 1 & 12 & 3 \\
\hline 5 & 8 & 7 & 6 & 15 \\
\hline \multirow{2}{*}{9} & \multirow{2}{*}{9} & 10 & 14 \\
\cline { 2 - 5 } & & 13 & 11 \\
\cline { 2 - 5 } & & &
\end{tabular}

\begin{tabular}{|c|c|c|c|c|}
\hline 1 & 2 & 3 & 5 & 9 \\
\hline 4 & 6 & 7 & 8 & 12 \\
\hline \multirow{2}{*}{10} & \multirow{2}{*}{10} & 11 & 13 \\
\cline { 2 - 5 } & & 14 & 15 \\
\cline { 3 - 5 } & & &
\end{tabular}

Fig. 9: An $(\mathbf{a}, \mathbf{r}, \mathbf{s})$-Selberg book on the left and an $(\mathbf{a}, \mathbf{r}, \mathbf{s})$-Young book on the right, where $\mathbf{a}=(1,2), \mathbf{r}=(1), \mathbf{s}=$ (2). The diagonal cells are shaded.

For $1 \leq i \leq m$, let $\lambda^{(i)}$ be an $\left(\mathbf{a}, r_{i}, s_{i}\right)$-staircase. We identify the $i$ th diagonal cells of $\lambda^{(1)}, \lambda^{(2)}, \ldots, \lambda^{(m)}$ for all $1 \leq i \leq n$. An $(\mathbf{a}, \mathbf{r}, \mathbf{s})$-Selberg book is a filling of $\left(\lambda^{(1)}, \ldots, \lambda^{(m)}\right)$ with $1,2, \ldots, N$ such that the integer in a non-diagonal cell is bigger than the integer in the diagonal cell of the same row and smaller than the integer in the diagonal cell of the same column. An (a, r, s)-Selberg book is given in Figure 9 Let $\mathrm{SB}(\mathbf{a}, \mathbf{r}, \mathbf{s})$ denote the set of $(\mathbf{a}, \mathbf{r}, \mathbf{s})$-Selberg books.

Now, for $1 \leq i \leq m$, let $\mu^{(i)}$ be a truncated $\left(\mathbf{a}, r_{i}, s_{i}\right)$-staircase. We identify the diagonal cells of $\mu^{(1)}, \mu^{(2)}, \ldots, \mu^{(m)}$ for each $1 \leq i \leq n$. A truncated $(\mathbf{a}, \mathbf{r}, \mathbf{s})$-Selberg book is a filling of $\left(\mu^{(1)}, \ldots, \mu^{(m)}\right)$ with $1,2, \ldots, N^{-}$such that the integer in a non-diagonal cell is bigger than the integer in the diagonal cell of the same row and smaller than the integer in the diagonal cell of the same column. Let $\mathrm{SB}^{-}(\mathbf{a}, \mathbf{r}, \mathbf{s})$ denote the set of truncated $(\mathbf{a}, \mathbf{r}, \mathbf{s})$-Selberg books.

There is a simple relation between $|\mathrm{SB}(\mathbf{a}, \mathbf{r}, \mathbf{s})|$ and $\left|\mathrm{SB}^{-}(\mathbf{a}, \mathbf{r}, \mathbf{s})\right|$.

Proposition 5.1. We have

$$
|\mathrm{SB}(\mathbf{a}, \mathbf{r}, \mathbf{s})|=\left|\mathrm{SB}^{-}(\mathbf{a}, \mathbf{r}, \mathbf{s})\right| \frac{N !}{\left(N^{-}\right) !} .
$$

By the same idea in Proposition 2.1, we obtain the following Proposition.

Proposition 5.2. We have

$$
\frac{n !}{\left(N^{-}\right) !}\left|\mathrm{SB}^{-}(\mathbf{a}, \mathbf{r}, \mathbf{s})\right|=\int_{0}^{1} \cdots \int_{0}^{1} \prod_{i=1}^{n} x_{i}^{r a_{i}}\left(1-x_{i}\right)^{s a_{i}} \prod_{1 \leq i<j \leq n}\left|x_{i}-x_{j}\right|^{m a_{i} a_{j}} d x_{1} \cdots d x_{n} .
$$

We define an $(\mathbf{a}, \mathbf{r}, \mathbf{s})$-Young book to be an $(\mathbf{a}, \mathbf{r}, \mathbf{s})$-Selberg book such that in each page entries are increasing from left to right in each row and from top to bottom in each column. An $(\mathbf{a}, \mathbf{r}, \mathbf{s})$-Young book is given in Figure 9 . Let $\mathrm{YB}(\mathbf{a}, \mathbf{r}, \mathbf{s})$ denote the set of $(\mathbf{a}, \mathbf{r}, \mathbf{s})$-Young books. We also define $\mathrm{SB}\left(\mathbf{a}, \mathbf{r}, \mathbf{s} ; d_{0}, d_{1}, \ldots, d_{n}\right)$ and $\operatorname{YB}\left(\mathbf{a}, \mathbf{r}, \mathbf{s} ; d_{0}, d_{1}, \ldots, d_{n}\right)$ to be, respectively, the set of $(\mathbf{a}, \mathbf{r}, \mathbf{s})$-Selberg books and the set of $(\mathbf{a}, \mathbf{r}, \mathbf{s})$-Young books whose diagonal entries $a_{1}, \ldots, a_{n}$ satisfy $d_{i}=a_{i+1}-a_{i}-1$ for $i=0,1,2, \ldots, n$, where $a_{0}=1$ and $a_{n+1}=N$.

There is a simple relation between $|\mathrm{SB}(\mathbf{a}, \mathbf{r}, \mathbf{s})|$ and $|\mathrm{YB}(\mathbf{a}, \mathbf{r}, \mathbf{s})|$. 


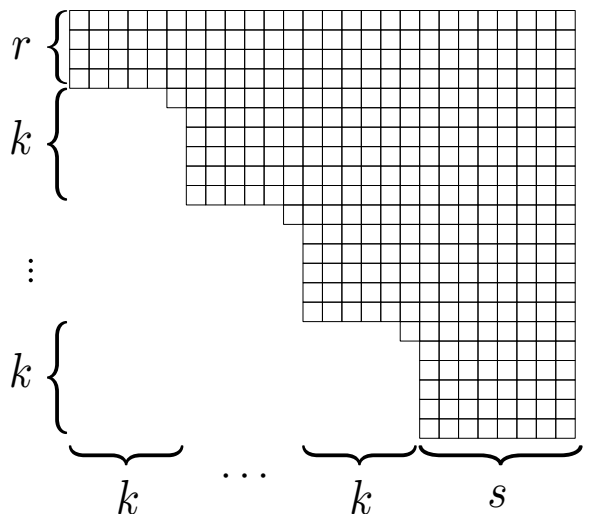

Fig. 10: The truncated shape $\lambda \backslash \mu$ for $\lambda=\left((k n+s)^{r+k n}\right)$ and $\mu=\left((k n)^{k-1}, k n-1,(k n-k)^{k-1}, k n-k-\right.$ $\left.1, \ldots, k^{k-1}, k-1\right)$.

\begin{tabular}{|c|c|c|c|c|c|c|c|c|c|c|c|c|c|c|c|c|}
\hline 1 & 2 & 3 & 4 & 5 & 6 & 10 & 11 & \multirow{7}{*}{$\Leftrightarrow$} & 1 & 2 & 3 & 4 & 5 & 6 & 10 & 11 \\
\hline \multirow{3}{*}{\multicolumn{2}{|c|}{7}} & & 8 & 12 & 14 & 17 & 20 & & & & 7 & 8 & 12 & 14 & 17 & 20 \\
\hline & & & 9 & 15 & 16 & 18 & 24 & & & & & 9 & 15 & 16 & 18 & 24 \\
\hline & & & 13 & 19 & 21 & 22 & 27 & & & & & 13 & 19 & 21 & 22 & 27 \\
\hline & & & & & 25 & 28 & & & & & & & 23 & 25 & 28 \\
\hline & & & & & & 26 & 29 & & & & & & & & 26 & 29 \\
\hline & & & & & & 30 & 31 & & & & & & & & 30 & 31 \\
\hline
\end{tabular}

Fig. 11: The correspondance between $\left(\left(k^{n}\right),(r),(s)\right)$-Young books and standard Young tableaux of truncated shape in Figure 10

Proposition 5.3. We have

$$
\left|\mathrm{SB}\left(\mathbf{a}, \mathbf{r}, \mathbf{s} ; d_{0}, d_{1}, \ldots, d_{n}\right)\right|=\left|\mathrm{YB}\left(\mathbf{a}, \mathbf{r}, \mathbf{s} ; d_{0}, d_{1}, \ldots, d_{n}\right)\right| \prod_{i=1}^{m} \frac{F\left(a+r_{i}+s_{i}\right)}{F\left(a_{1}\right) \cdots F\left(a_{n}\right) F\left(r_{i}\right) F\left(s_{i}\right)}
$$

where $F(k)=1 ! 2 ! \cdots(k-1) !$

If $\mathbf{a}=(k, k, \ldots, k)$, then we can evaluate $|\mathrm{YB}(\mathbf{a}, \mathbf{r}, \mathbf{s})|$.

Corollary 5.4. Let $\mathbf{a}=(k, k, \ldots, k)$. Then

$$
\begin{aligned}
|\mathrm{YB}(\mathbf{a}, \mathbf{r}, \mathbf{s})|=\frac{2^{n}((k r+k s+1) n+}{} & \left.k^{2} m\left(\begin{array}{c}
n \\
2
\end{array}\right)+\sum_{i=1}^{m} r_{i} s_{i}\right) ! \\
n ! & \prod_{i=1}^{m} \frac{F(k)^{n} F\left(r_{i}\right) F\left(s_{i}\right)}{F\left(k n+r_{i}+s_{i}\right)} \\
& \times \prod_{j=1}^{n} \frac{\left(j k^{2} m\right) ! !\left(2 k r+(j-1) k^{2} m\right) ! !\left(2 k s+(j-1) k^{2} m\right) ! !}{\left(k^{2} m\right) ! !\left(2 k r+2 k s+2+(n+j-2) k^{2} m\right) ! !} .
\end{aligned}
$$

If $\mathbf{a}=\left(k^{n}\right), \mathbf{r}=(r), \mathbf{s}=(s)$, then by replacing the each diagonal cell with a $1 \times 1$ cell located at the northeast corner of the diagonal cell, we can consider an $(\mathbf{a}, \mathbf{r}, \mathbf{s})$-Young book as a standard Young tableau of truncated shape $\lambda \backslash \mu$ shown in Figure 10 See Figure 11 for the illustration of this correspondence. Thus we get the following corollary. 
Corollary 5.5. The number of standard Young tableaux of truncated shape in Figure 10 is equal to

$$
\begin{aligned}
& \frac{2^{n}\left((k r+k s+1) n+k^{2}\left(\begin{array}{c}
n \\
2
\end{array}\right)+r s\right) !}{n !} \frac{F(k)^{n} F(r) F(s)}{F(k n+r+s)} \\
& \quad \times \prod_{j=1}^{n} \frac{\left(j k^{2}\right) ! !\left(2 k r+(j-1) k^{2}\right) ! !\left(2 k s+(j-1) k^{2}\right) ! !}{\left(k^{2}\right) ! !\left(2 k r+2 k s+2+(n+j-2) k^{2}\right) ! !}
\end{aligned}
$$

where $F(n)=1 ! 2 ! \ldots(n-1)$ !.

Panova [Pan12] found a formula for the number of standard Young tableaux of truncated shape $\left(n^{m}\right) \backslash(k-$ $1, k-2, \ldots, 1)$ and $\left(n^{m}\right) \backslash\left(k^{k-1}, k-1\right)$. Both of these truncated shapes are special cases of the truncated shape in Corollary 5.5 .

\section{Final remarks}

The original motivation of this paper was to find a combinatorial proof of the Selberg integral formula when $r=\alpha-1, s=\beta-1$ and $m=2 \gamma$ are nonnegative integers. We can achieve this goal if we solve the following two problems.

Problem 6.1. Find a combinatorial proof of Theorem 3.3 .

Problem 6.2. Find a combinatorial proof of Corollary 3.5

One can consider Young books of shape $\left(\lambda^{(1)}, \ldots, \lambda^{(m)}\right)$ for shifted Young diagrams $\lambda^{(i)}$ with the same number of rows. However, in this case we do not seem to have a nice factorization formula. For instance, the number of Young books of shape

$$
((6,2,1),(5,4,1),(5,2,1),(4,2,1))
$$

is equal to

$$
2^{4} \cdot 3 \cdot 5^{2} \cdot 7 \cdot 17 \cdot 19 \cdot 23 \cdot 1649819
$$

\section{References}

[FW08] P. Forrester and S. Warnaar. The importance of the selberg integral. Bull. Amer. Math. Soc., 45:489-534, 2008.

[Pan12] Greta Panova. Tableaux and plane partitions of truncated shapes. Advances in Applied Mathematics, 49(3-5):196-217, 2012.

[Pos09] A. Postnikov. Permutohedra, associahedra, and beyond. Int. Math. Res. Not., 2009(6):1026-1106, 2009.

[Sel44] A. Selberg. Remarks on a multiple integral. Norsk Mat. Tidsskr, 26:71-78, 1944.

[Sta11] R. Stanley. Enumerative combinatorics. Vol. 1. Cambridge University Press, Cambridge, 2011. 